\title{
Extension of the Nambu-Jona-Lasinio model predictions at high densities and temperatures using an implicit regularization scheme
}

\author{
R. L. S. Farias, ${ }^{1}$ G. Dallabona, ${ }^{2}$ G. Krein, ${ }^{3}$ and O. A. Battistel ${ }^{4}$ \\ ${ }^{1}$ Departamento de Física Teórica, Universidade do Estado do Rio de Janeiro, Rio de Janeiro, RJ 20559-900, Brazil \\ ${ }^{2}$ Departamento de Ciências Exatas, Universidade Federal de Lavras, Cx. Postal 37, 37200-000, Lavras, MG, Brazil \\ ${ }^{3}$ Instituto de Física Teórica, Universidade Estadual Paulista, Rua Pamplona 145, São Paulo, SP 01405-900, Brazil \\ ${ }^{4}$ CBPF, Centro Brasileiro de Pesquisas Fisicas Rua Xavier Sigaud 150, 22290-180 Urca Rio de Janeiro, RJ, Brazil
}

(Received 23 October 2007; published 9 June 2008)

\begin{abstract}
Traditional cutoff regularization schemes of the Nambu-Jona-Lasinio model limit the applicability of the model to energy-momentum scales much below the value of the regularizing cutoff. In particular, the model cannot be used to study quark matter with Fermi momenta larger than the cutoff. In the present work, an extension of the model to high temperatures and densities recently proposed by Casalbuoni, Gatto, Nardulli, and Ruggieri is used in connection with an implicit regularization scheme. This is done by making use of scaling relations of the divergent one-loop integrals that relate these integrals at different energy-momentum scales. Fixing the pion decay constant at the chiral symmetry breaking scale in the vacuum, the scaling relations predict a running coupling constant that decreases as the regularization scale increases, implementing in a schematic way the property of asymptotic freedom of quantum chromodynamics. If the regularization scale is allowed to increase with density and temperature, the coupling will decrease with density and temperature, extending in this way the applicability of the model to high densities and temperatures. These results are obtained without specifying an explicit regularization. As an illustration of the formalism, numerical results are obtained for the finite density and finite temperature quark condensate and applied to the problem of color superconductivity at high quark densities and finite temperature.
\end{abstract}

DOI: 10.1103/PhysRevC.77.065201

PACS number(s): 12.39.-x, 24.85.+p, 21.65.-f

\section{INTRODUCTION}

The Nambu-Jona-Lasinio (NJL) [1] model has been the prototype model for studying chiral symmetry restoration in hadronic matter at finite baryon densities $\rho_{B}$ and finite temperatures $T$. Since the earlier applications of the model at high $\rho_{B}$ and $T$ [2,3], an extensive and important body of work has been done in this direction-for reviews and a comprehensive list of references see Refs. [4-10]. Because the model is nonrenormalizable, the high momentum part of the model has to be regularized in a phenomenological way. The common practice has been to regularize the divergent loop amplitudes with a three-dimensional momentum cutoff $\Lambda \sim 1 \mathrm{GeV}$, which also sets the energy-momentum scale for the validity of the model. That is, the model cannot be used for studying phenomena involving momenta running in loops larger than $\Lambda$. In particular, the model cannot be used to study quark matter at high densities $\rho_{B} \sim k_{F}^{3}$ with $k_{F}>\Lambda$, where $k_{F}$ is the quark Fermi momentum. One of the symptoms of this problem is the prediction of vanishing superconducting gaps at high baryon densities, a feature of the model that is solely caused by the use of a regularizing momentum cutoff $\Lambda$ of the divergent loop integrals [11-13].

Recently, we [14] presented an alternative to the cutoff regularization of the NJL model, in which the one-loop integrals are reorganized through mathematical identities in such a way that finite integrals become separated from density-independent divergent integrals. The finite integrals are integrated without imposing any restriction on the integration momenta, and the divergent integrals are related to physical quantities at the dynamical chiral symmetry breaking in vacuum. As a result, instead of a vanishing gap like that in the cutoff regularization, one obtains a finite gap that grows with density. However, this does not mean that the model can be used at arbitrarily high densities. The point is that any implicit regularized integral contains an implicit scale, the regularization scale. When such an integral is fitted to a physical quantity in vacuum, we are implicitly also fitting this scale. Now, in a loop integral at nonzero density and/or temperature, the vacuum part is determined by the regularization scale, and the finite integrals are governed by the chemical potential and temperature. When these scales are very different, one obtains nonsense results. Nevertheless, this mismatch of scales is less problematic in the implicit regularization scheme than in any other cutoff regularization scheme, in the sense that one can go to much higher densities and temperatures before the problem shows up.

The root of the problem is of course the nonrenormalizability of the model. In a renormalizable theory, all the dependence on the cutoff can be removed in favor of running physical parameters, such as the coupling constants of QED and QCD. The running is given by the renormalization group equations of the theory and is controlled by an energy scale that is adjusted to the scale of the experimental conditions under consideration. In a recent publication, Casalbuoni et al. [11] introduced the concept of a running coupling constant for the NJL model to extend the applicability of the model to high density. The arguments are based on making the cutoff density dependent, using an analogy with an ordinary solid. In an ordinary solid, there is a natural maximum phonon frequency - the Debye frequency - that increases as the lattice spacing gets smaller. 
In quark matter, as the density of quarks increases, the quarks get closer together, and therefore the ultraviolet cutoff $\Lambda$ of the NJL model should be allowed to increase correspondingly. The cutoff can be changed consistently without spoiling the predictions of the model for the chiral properties of the vacuum if the four-fermion coupling constant $G$ of the model is allowed to change with the cutoff. In Ref. [11], the cutoff dependence of $G$ is obtained from the joint consideration of the divergent expressions for the pion decay constant $f_{\pi}$ and the gap equation for the constituent quark mass $M$ : fixing the value of $f_{\pi}$ at $93 \mathrm{MeV}$, the divergent expression of $f_{\pi}$ (in the chiral limit), regulated with a cutoff $\Lambda$, leads to a constituent quark mass $M$ that is $\Lambda$ dependent. The use of this $M=M(\Lambda)$ in the gap equation gives rise to a coupling $G$ that runs with $\Lambda$. Moreover, the running of the coupling is such that $G(\Lambda)$ decreases with increasing $\Lambda$. This is certainly physically motivated and is also in accord with the interpretation $[2,15]$ that the cutoff in the NJL model simulates - albeit in a crude way-the property of asymptotic freedom of QCD, in the sense that the coupling between quarks decreases as higher momentum scales are probed. A similar suggestion was made by Shakin and collaborators [16] in studies of hadronic correlation functions. They found that results in qualitative accord with lattice QCD calculations can be obtained once a significant temperature dependence of the cutoff and of the coupling $G$ is allowed.

The aim of the present paper is to show that it is possible to extend the applicability of the NJL model to high densities and temperatures without the use of an explicit regularization of divergences. The basic motivation for avoiding an explicit regularization, such as the commonly used three- or four-momentum cutoff, Pauli-Villars, or proper-time regularizations, is that these lead to global and gauge symmetry violations and to the breaking of causality and unitarity. Although in many situations these problems do not have great influences on the final numerical results, there are situations where they do have drastic consequences, as in studies of correlation functions. Arguments have been used and tricks invented to circumvent such problems, but no satisfactory solution has been found-for a good discussion on these issues, see, for example, Refs. [17-19].

The implicit regularization scheme was originally proposed in Ref. [20] and has been used in different contexts [21,22], including applications to the NJL model [14,23-25]. At the one-loop approximation, there appears only two divergent integrals: one is quadratically divergent and the other is logarithmically divergent. Once the divergent integrals are assumed to be implicitly regulated, i.e., regulated without the specification of an explicit regulator, they will depend implicitly on a momentum scale, which we denote by $\Lambda$. These integrals satisfy well-defined scaling relations, in that the integrals at some mass scale can be related to a combination of the same integrals at some another, arbitrary scale. These scaling relations allow one to express the divergent parts of the amplitudes at finite temperature and density in terms of their counterparts at zero temperature and density. Moreover, fixing $f_{\pi}$ in the vacuum, one can derive a scaling relation for the four-fermion coupling $G$ which gives a running $G(\Lambda)$ similar to that found in Ref. [11]. All this is achieved, we reiterate, without specifying an explicit regularization scheme.

In the next section, we review the main aspects of the implicit regularization scheme [14,20,21,23-25] that are most relevant to the present paper. We start by specifying the Lagrangian density we are going to use, and then we explain the scaling relations and their use in obtaining the solution of the gap equation within this scheme. To make contact with results of the literature, we solve numerically this gap equation at finite temperature and density and show the results for the quark condensate as a function of temperature $T$ and quark chemical potential $\mu$. In Sec. III, we use the scaling relations to obtain the running of the coupling $G$ of the model and compare results with those in Ref. [11]. In Sec. IV, we illustrate the use of our results for obtaining the critical temperature as a function of quark chemical potential for the spin-0 two-flavor color superconducting (2SC) gap. Our conclusions and outlook are presented in Sec. V.

\section{CALCULATIONAL STRATEGY TO HANDLE THE DIVERGENCES}

For the purposes of the present work, it is sufficient to consider the simplest version of the model specified by the two-flavor SU(2) Lagrangian density

$$
\mathcal{L}_{\mathrm{NJL}}=\bar{\psi}\left(i \not \partial-m_{0}\right) \psi+G\left[(\bar{\psi} \psi)^{2}-\left(\bar{\psi} \gamma^{5} \vec{\tau} \psi\right)^{2}\right] .
$$

Here $\psi$ is the quark field operator (with color and flavor indices suppressed), $m_{0}$ is the current-quark mass matrix. At the oneloop approximation, the gap equation at finite temperature $T$ and quark chemical potential $\mu$ is given by (for simplicity we work in the chiral limit $m_{0}=0$ )

$$
M=48 G M T \sum_{n} \int \frac{d^{3} k}{(2 \pi)^{3}} \frac{1}{\left(i \omega_{n}+\mu\right)^{2}+E(k)^{2}},
$$

with $E(k)=\sqrt{k^{2}+M^{2}}$, and $\omega_{n}=(2 n+1) \pi T, n=0, \pm 1$, $\pm 2, \ldots$, being the Matsubara frequencies. Performing the sum, one obtains

$$
M=48 G M\left[i I_{\text {quad }}(M)-I(T, \mu)\right],
$$

where

$$
I_{\text {quad }}\left(M^{2}\right)=\int \frac{d^{4} k}{(2 \pi)^{4}} \frac{1}{k^{2}-M^{2}},
$$

and

$$
I(T, \mu)=\int \frac{d^{3} k}{(2 \pi)^{3}} \frac{\left[n_{-}(k)+n_{+}(k)\right]}{2 E(k)},
$$

with $n_{ \pm}(k)$ being the quark and antiquark Fermi-Dirac distributions

$$
n_{ \pm}(k)=\frac{1}{e^{[E(k) \pm \mu] / T}+1} .
$$

While $I(T, \mu)$ is finite, $I_{\text {quad }}\left(M^{2}\right)$ is divergent. The divergent integral is an element of the systematization adopted in the calculational strategy [26] which we use in the present work. In four-dimensional one-loop calculations within the context of theories and models where the divergence degree is not higher 
than cubic, which is the case of the NJL model, there also appears, besides $I_{\text {quad }}\left(M^{2}\right)$, the basic logarithmically divergent object

$$
I_{\log }\left(M^{2}\right)=\int \frac{d^{4} k}{(2 \pi)^{4}} \frac{1}{\left(k^{2}-M^{2}\right)^{2}} .
$$

In the NJL model, $I_{\text {quad }}\left(M^{2}\right)$ is related to the constituent quark mass, as shown in Eq. (3), whereas $I_{\log }\left(M^{2}\right)$ is related to the pion decay constant in vacuum-see Eq. (22).

The traditional procedure for treating the divergent amplitudes in nonrenormalizable models is the introduction of a regularization distribution in all loop integrals in order to render them convergent. In the context of the NJL model, with rare exceptions - such as in Refs. [27-29] - the finite integral $I\left(T, \mu_{B}\right)$ containing the Fermi-Dirac distributions is also regularized. Because of the nonrenormalizable character of the model, the regularization introduced in this way cannot be removed along the calculations, and therefore the predictions become associated with the chosen regularization. In practice, the amplitudes become functions of the regularization distribution parameters, and consequently the energy-momentum dependence of the amplitudes, as emerging from the Feynman rules, are modified. As is well-known, this leads to violation of fundamental symmetries such as those associated to space-time homogeneity or to the introduction of nonphysical thresholds that break unitarity.

Having this in mind, an alternative strategy to handling the divergences typical of the perturbative calculations in quantum field theory (QFT) has been developed in Ref. [20]. The main idea is to avoid the critical step of the explicit evaluation of a divergent integral. The finite and divergent parts are separated by using a convenient representation for the propagators in which all the dependence on the physical momenta is located in finite integrals that are not affected by regularization, thus avoiding contamination with nonphysical behaviors. The divergent parts obtained in this way are reduced to only a small number of basic objects after the adoption of a set of properties for purely divergent integrals, denominated consistency relations (CR), which are dictated by the requirements of symmetry preservation and elimination of ambiguities [7]. The remaining divergent objects need not be solved explicitly; in renormalizable theories, they are completely absorbed in renormalization of physical parameters, and in nonrenormalizable models they can be directly adjusted to physical quantities.

There are many advantages to adopting the procedure just described, but two of them can be immediately noted: the treatment for the divergences is universal, and the energymomentum dependence of the amplitudes is not modified. Besides, a connection with traditional procedures can be made. In particular, all results can be mapped to the ones obtained within the context of dimensional regularization (DR), in situations where this method applies. On the other hand, the connection with regularizations based on regularizing distributions requires some care in the case of nonrenormalizable models, because the effects of regularization on finite integrals are removed in our strategy. In this sense, it is important to say that the prescription described above, which has been applied in many problems involving divergences in QFT [21], represents a particular prescription to handle the amplitudes having divergences. Therefore, it should be considered a procedure to make predictions with nonrenormalizable models in the same way as with any other regularization prescription. However, the important point to be noted is that, by construction, many of the problems intrinsic to the traditional methods have been circumvented, such as violations of symmetry relations among Green's functions, ambiguities, nonphysical thresholds, and scale invariance breaking. The convenience or usefulness of the method for the treatment of nonrenormalizable models should be judged by the consistency of its phenomenological implications.

At one-loop order, only two divergent integrals, $I_{\text {quad }}\left(M^{2}\right)$ and $I_{\log }\left(M^{2}\right)$, need to be eliminated by some phenomenological adjustment. We first note that they are not independent quantities, that is, they obey two scaling relations [30]

$$
\begin{aligned}
I_{\text {quad }}\left(M^{2}\right)= & I_{\text {quad }}\left(\lambda^{2}\right)+\left(M^{2}-\lambda^{2}\right) I_{\log }\left(\lambda^{2}\right) \\
& +\frac{i}{(4 \pi)^{2}}\left[M^{2}-\lambda^{2}-M^{2} \log \left(\frac{M^{2}}{\lambda^{2}}\right)\right], \\
I_{\log }\left(M^{2}\right)= & I_{\log }\left(\lambda^{2}\right)-\frac{i}{(4 \pi)^{2}} \log \left(\frac{M^{2}}{\lambda^{2}}\right),
\end{aligned}
$$

where $\lambda^{2}$ is the arbitrary mass scale adopted in the separation of finite and divergent parts. These relations allow us to relate the basic divergent quantities in two different mass scales, as we shall see in a moment. The imposition of scale independence implies two properties for these objects:

$$
\begin{aligned}
\frac{\partial I_{\text {quad }}\left(\lambda^{2}\right)}{\partial \lambda^{2}} & =I_{\log }\left(\lambda^{2}\right), \\
\frac{\partial I_{\log }\left(\lambda^{2}\right)}{\partial \lambda^{2}} & =-\frac{i}{(4 \pi)^{2}} \frac{1}{\lambda^{2}} .
\end{aligned}
$$

It is important to note that only finite quantities have been differentiated. The above properties work like requirements that must be imposed in regularizations in order to maintain the scaling properties of the amplitudes. The above referred properties can be understood within the context of regularizations. For this purpose, we assume that each integral is regularized through an unspecified distribution $f(k / \Lambda)$, where $\Lambda$ is a parameter with the dimensions of momentum such that

$$
\begin{aligned}
I_{\text {quad }}\left(M^{2}\right) & =\int \frac{d^{4} k}{(2 \pi)^{4}} \frac{f(k / \Lambda)}{k^{2}-M^{2}}, \\
I_{\log }\left(M^{2}\right) & =\int \frac{d^{4} k}{(2 \pi)^{4}} \frac{f(k / \Lambda)}{\left(k^{2}-M^{2}\right)^{2}} .
\end{aligned}
$$

Differentiating $I_{\text {quad }}\left(M^{2}\right)$, relation (10) follows immediately. By differentiating Eq. (13), on the other hand, we obtain

$$
\frac{\partial I_{\log }\left(M^{2}\right)}{\partial M^{2}}=2 \int \frac{d^{4} k}{(2 \pi)^{4}} \frac{f(k / \Lambda)}{\left(k^{2}-M^{2}\right)^{3}} .
$$

Since the integral is finite and assuming the existence of the limit $\lim _{\Lambda \rightarrow \infty} f(k / \Lambda)=1$, one can extract the regulating distribution and perform the integration to obtain Eq. (11). This last point illustrates the basic difference between our prescription and the traditional ones. 
The fact that the parameter $\Lambda$ plays the role of a cutoff can be made clear even in the case of an implicit regularization. That is, it is possible to make the $\Lambda$ dependence explicit without specifying the regulating function. This can be done by defining the dimensionless ratio $M_{\Lambda}=M / \Lambda$ and writing $I_{\text {quad }}\left(M^{2}\right)$ and $I_{\log }\left(M^{2}\right)$ in terms of dimensionless integrals $J_{\text {quad }}\left(M_{\Lambda}^{2}\right)$ and $J_{\log }\left(M_{\Lambda}^{2}\right)$ as

$$
\begin{aligned}
I_{\text {quad }}\left(M^{2}\right) & =\Lambda^{2} J_{\text {quad }}\left(M_{\Lambda}^{2}\right), \\
I_{\log }\left(M^{2}\right) & =J_{\log }\left(M_{\Lambda}^{2}\right),
\end{aligned}
$$

with

$$
\begin{aligned}
J_{\text {quad }}\left(M_{\Lambda}^{2}\right) & =\int \frac{d^{4} u}{(2 \pi)^{4}} \frac{f(u)}{u^{2}-M_{\Lambda}^{2}}, \\
J_{\log }\left(M_{\Lambda}^{2}\right) & =\int \frac{d^{4} u}{(2 \pi)^{4}} \frac{f(u)}{\left(u^{2}-M_{\Lambda}^{2}\right)^{2}},
\end{aligned}
$$

where the integration variable $u$ is dimensionless. The integrals $J_{\text {quad }}\left(M_{\Lambda}^{2}\right)$ and $J_{\log }\left(M_{\Lambda}^{2}\right)$ obey scaling relations completely similar to those in Eqs. (8) and (9), as they should. A general parametrization for these integrals, obeying the properties of Eqs. (10) and (11), can be constructed and written as

$$
\begin{aligned}
i(4 \pi)^{2} J_{\log }\left(M_{\Lambda}^{2}\right) & =\ln \left(M_{\Lambda}^{2}\right)+c_{1}, \\
i(4 \pi)^{2} J_{\text {quad }}\left(M_{\Lambda}^{2}\right) & =M_{\Lambda}^{2} \ln \left(M_{\Lambda}^{2}\right)-M_{\Lambda}^{2}+c_{2} .
\end{aligned}
$$

It should be clear that if a regularization distribution does not furnish the above general form, the scaling properties of the amplitudes can be broken. In principle, different (consistent) regularizations should differ only by the values of the constants $c_{1}$ and $c_{2}$.

After this review on the general procedure to handle the divergences, let us turn our attention to its application to the NJL model. First, we have that the vacuum quark condensate is related to the scalar one-point function, which can be written as

$$
\langle\bar{\psi} \psi\rangle_{0}=-12 M_{0} i I_{\text {quad }}\left(M_{0}^{2}\right),
$$

where $M_{0}$ is the mass obtained by solving the gap equation in vacuum. On the other hand, the pion decay constant $f_{\pi}$ can be related to the axial-pseudoscalar two-point function such that

$$
f_{\pi}^{2}=-12 M_{0}^{2} i I_{\log }\left(M_{0}^{2}\right) .
$$

The point now is that one can express the temperatureand density-dependent gap equation (3) for quark mass $M$ in terms of these vacuum quantities. This is so because in general the scaling relations (8) and (9) can be used to isolate vacuum contributions from temperature- and densitydependent contributions in expressions for physical quantities; that is, it is possible to write $I_{\text {quad }}\left(M^{2}\right)$ and $I_{\log }\left(M^{2}\right)$ in terms of $I_{\text {quad }}\left(M_{0}^{2}\right)$ and $I_{\log }\left(M_{0}^{2}\right)$. Using the scaling relation in Eq. (3), we get

$$
\begin{aligned}
M= & 48 G M\left\{-\frac{\langle\bar{\psi} \psi\rangle_{0}}{12 M_{0}}-\left(M^{2}-M_{0}^{2}\right) \frac{f_{\pi}^{2}}{12 M_{0}^{2}}\right. \\
& \left.-\frac{1}{(4 \pi)^{2}}\left[M^{2}-M_{0}^{2}-M^{2} \log \left(\frac{M^{2}}{M_{0}^{2}}\right)\right]-I(T, \mu)\right\},
\end{aligned}
$$

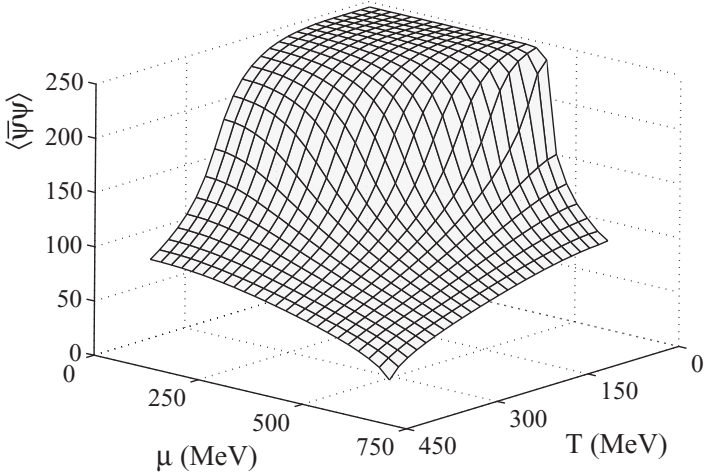

FIG. 1. One-flavor quark condensate $\langle\bar{\psi} \psi\rangle$ as function of temperature $T$ and quark chemical potential $\mu$.

where we have made use of Eqs. (21) and (22). The corresponding expression for the case $m_{0} \neq 0$ is a little more complicated than the one shown in Eq. (23), since the expression for $f_{\pi}^{2}$ contains, in addition to $I_{\log }$, a finite integral involving the pion mass.

For illustrative purposes, we present in Fig. 1 the quark condensate as a function of temperature $T$ and quark chemical potential $\mu$; the results here are obtained solving the gap equation with $m_{0}=5.5 \mathrm{MeV}, G=4.9 \times 10^{-6} \mathrm{MeV}^{-2}$, and the fitting mass $M_{0}=312 \mathrm{MeV}$. As seen in Fig. 1, this implicit regularization scheme gives the expected reduction of the quark condensate in medium. In addition, this reduction is in qualitative agreement with the result obtained with traditional cutoff regularization schemes; see, for example, Fig. 26 of Ref. [4]. In the chiral limit, the order of the phase transition is first order. It is also worth mentioning that with these same parameters, one is able to obtain good values for the $\pi$ - and $\sigma$-meson masses [25].

Of course, the point of the exercise here was not only to show that this different way of handling the divergent integrals gives results in accord with the expected phenomenology. What should be noted here is that once we have eliminated $I_{\text {quad }}\left(M_{0}^{2}\right)$ and $I_{\log }\left(M_{0}^{2}\right)$ in favor of $\langle\bar{\psi} \psi\rangle_{0}$ and $f_{\pi}$, we have kept $\Lambda$ implicitly fixed. If one wants to change $\Lambda$ without changing the low-energy results, $G$ has to run with $\Lambda$, as in Ref. [11]. The point here is that one can implement this using the scaling relations (8) and (9), which are independent of an explicit regularization. This will be done in the next section.

\section{RUNNING OF THE COUPLING}

Fixing $f_{\pi}=93 \mathrm{MeV}$ and using the expression of $f_{\pi}$ in terms of $I_{\log }$ at the scales $(M, \Lambda)$ and $\left(M_{0}, \Lambda_{0}\right)$, one obtains $M=M(\Lambda)$ as the solution of the transcendental equation

$$
M^{2}=M_{0}^{2} \frac{\Lambda^{2}}{\Lambda_{0}^{2}} \exp \left[\frac{4 \pi^{2}}{3} \frac{f_{\pi}^{2}}{M^{2}}\left(\frac{M^{2}}{M_{0}^{2}}-1\right)\right]
$$

Using the value of $\langle\bar{\psi} \psi\rangle_{0}$ at the reference scale $\left(M_{0}, \Lambda_{0}\right)$, and using the scaling relation (8) to obtain $I_{\text {quad }}$ at $(M, \Lambda)$ in the 


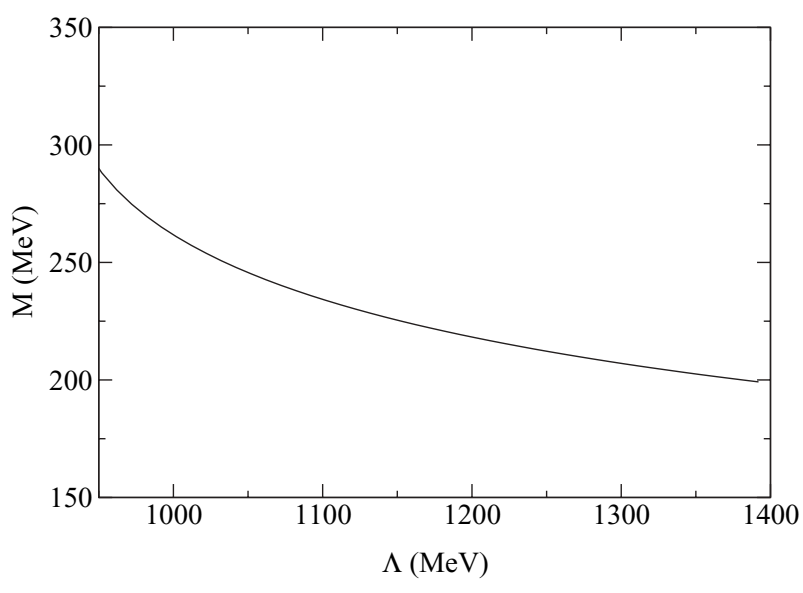

FIG. 2. Constituent quark mass $M$ as a function of $\Lambda$.

gap equation, one obtains $G=G(\Lambda)$ as

$$
\begin{aligned}
{\left[48 \Lambda^{2} G(\Lambda)\right]^{-1} } & =\frac{-\langle\bar{\psi} \psi\rangle_{0}}{12 M_{0} \Lambda_{0}^{2}}-\left(\frac{M^{2}}{\Lambda^{2}}-\frac{M_{0}^{2}}{\Lambda_{0}^{2}}\right) \frac{f_{\pi}^{2}}{12 M_{0}^{2}}-\frac{1}{(4 \pi)^{2}} \\
& \times\left[\frac{M^{2}}{\Lambda^{2}}-\frac{M_{0}^{2}}{\Lambda_{0}^{2}}-\frac{M^{2}}{\Lambda^{2}} \log \left(\frac{M^{2} \Lambda_{0}^{2}}{\Lambda^{2} M_{0}^{2}}\right)\right],
\end{aligned}
$$

where it is understood that $M=M(\Lambda)$, as given by Eq. (24).

This is our main result in the present paper. It is an interesting result, in that it was obtained without specifying any explicit regularization; only very general scaling relations of the divergent integrals were used. In this sense, the result seems very general and robust. Once a temperature and density dependence for $\Lambda$ is specified, $G$ becomes also temperature and density dependent.

In Fig. 2, we present the numerical results for the $\Lambda$ dependence of $M$, as obtained from the solution of Eq. (24) after solving the gap equation. The constituent mass decreases as $\Lambda$ is increased. If $G$ were kept fixed, $M$ would increase, of course. But $G$ decreases with $\Lambda$, as shown in Fig. 3, and the net effect is that $M$ decreases. These results for $M=M(\Lambda)$ and $G=G(\Lambda)$ are in qualitative agreement with the results of Ref. [11].

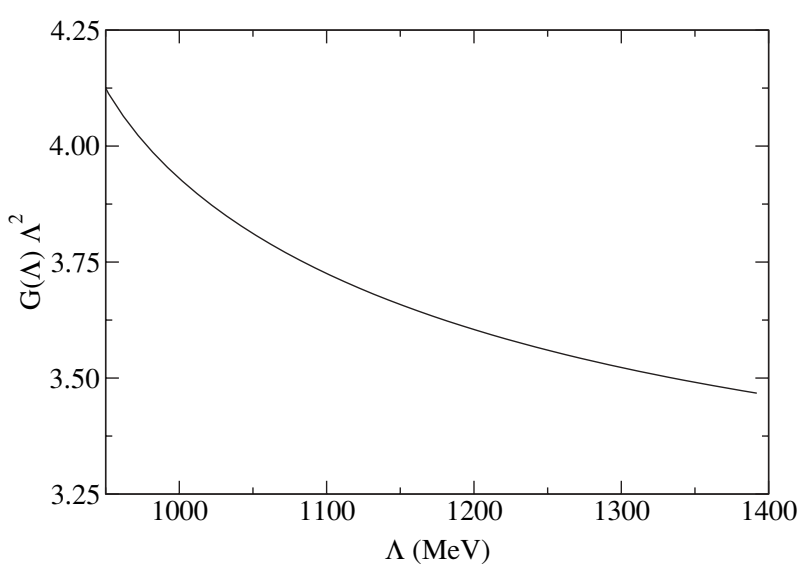

FIG. 3. Running of the coupling $G(\Lambda)$.
The specification of a density and temperature dependence for $\Lambda$ might seem a little arbitrary. But this need not be the case, because the entire line of arguments can be turned around: instead of fixing $f_{\pi}$ at some value, one could postulate in a physically motivated way a running behavior for $G=G(\Lambda)$ and work backward. What would change in this case? First, using the postulated $G(\Lambda)$ in the gap equation, one would obtain a corresponding $M(\Lambda)$. When this $M(\Lambda)$ is replaced in the expression for $f_{\pi}$, in general $f_{\pi}$ will be also $\Lambda$ dependent, but this $\Lambda$ dependence would be very weak, since the integral for $f_{\pi}$ is only logarithmically divergent. In this way, the chiral physics in vacuum would be maintained. This is very interesting, since one could use the predicted density and temperature running of the QCD coupling constant for $G$ and in this way fit the density and temperature dependence of $\Lambda$. In this way, one would be modeling, admittedly in a crude way, the asymptotic freedom of QCD in the NJL model.

In closing this section, we reiterate that the purpose for making $G$ to run with $\Lambda$ is to extend the applicability of the model to high densities and temperatures. At high densities and temperatures, high momentum components are present in the system, and a cutoff of the order of the chiral symmetry breaking scale invalidates the use of the model in such situations. To illustrate the use of the extension in practice, we consider in the next section color superconductivity in high-density quark matter.

\section{COLOR SUPERCONDUCTIVITY}

For reviews and a comprehensive list of references on the subject of color superconductivity, see Refs. [31-38]. Since the aim here is to illustrate the formalism, we simplify matters by using the same Lagrangian density as used above; although for obtaining a better phenomenological description of both chiral symmetry breaking and color superconductivity, a more general four-fermion Lagrangian should be used [12,13,39]. At the one-loop level, the self-consistent equation for the superconducting gap is given by (we use the same letter $G$ to denote both the diquark coupling here and the quark-antiquark coupling in the last section)

$$
1=16 G \int \frac{d^{4} k}{(2 \pi)^{4}} \frac{i}{k_{0}^{2}-(k+\mu)^{2}-\Delta^{2}}+(\mu \rightarrow-\mu) .
$$

The integrals above are divergent. The application of the implicit regularization scheme to this problem proceeds as follows [14]. Instead of introducing a cutoff in the integrals, the integrands are assumed to be implicitly regularized and then manipulated in such a way that divergences are isolated in $\mu$-independent divergent integrals. These divergent integrals can be related to the divergent integrals $I_{\text {quad }}$ and $I_{\log }$ of the problem of chiral symmetry breaking in vacuum through the use of the scaling relations discussed above. From the manipulation of the integrand in Eq. (26), finite integrals also result, and these are integrated without imposing any restriction to their integrands. Initially we consider $T=0$. In this case, the equation for the superconducting gap is 


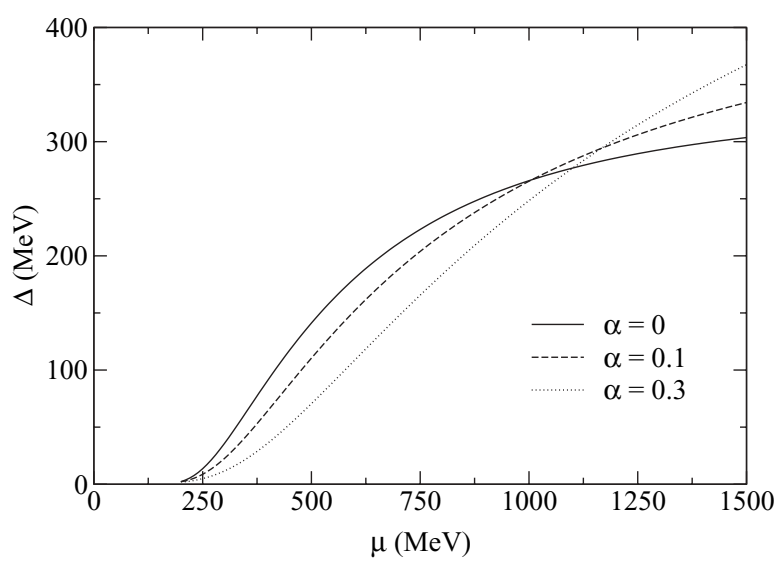

FIG. 4. Zero temperature superconducting gap as a function of quark chemical potential for three different values of $\alpha$ in Eq. (28).

given by

$$
\begin{aligned}
1= & 2 G(\Lambda) \Lambda^{2}\left\{-\frac{4}{3} \frac{\langle\bar{\psi} \psi\rangle_{0}}{M_{0} \Lambda_{0}^{2}}-\frac{4}{3}\left(\frac{\Delta^{2}}{\Lambda^{2}}-\frac{M_{0}^{2}}{\Lambda_{0}^{2}}\right) \frac{f_{\pi}^{2}}{M_{0}^{2}}\right. \\
& -\frac{1}{\pi^{2}}\left[\frac{\Delta^{2}}{\Lambda^{2}}-\frac{M_{0}^{2}}{\Lambda_{0}^{2}}-\frac{\Delta^{2}}{\Lambda^{2}} \log \left(\frac{\Delta^{2} \Lambda_{0}^{2}}{\Lambda^{2} M_{0}^{2}}\right)+2 \frac{\mu^{2}}{\Lambda^{2}}\right] \\
& \left.+\frac{\mu^{2}}{\Lambda^{2}}\left[\frac{8}{3} \frac{f_{\pi}^{2}}{M_{0}^{2}}-\frac{2}{\pi^{2}} \log \left(\frac{\Delta^{2} \Lambda_{0}^{2}}{\Lambda^{2} M_{0}^{2}}\right)\right]\right\},
\end{aligned}
$$

where we used the manipulations of the integrand in Eq. (26) as explained above and shown explicitly in Ref. [14].

One very interesting result of the application of the implicit regularization to the problem of color superconductivity is that, as shown with greater detail in Ref. [14], the superconducting gap as a function of $\mu$ does not vanish at $\mu \simeq \Lambda$, as happens with the traditional cutoff schemes. The numerical result for $\Delta$ is shown by the solid line in Fig. 4. This is interesting because a nonvanishing gap at high quark densities is predicted by QCD [40]; see also the reviews in Refs. [31-38]. Of course, if the implicit regularization scale $\Lambda$ is kept fixed, the use of the model at high densities is questionable. However, we are able to make explicit the $\Lambda$ dependence in the gap equation (and in other physical quantities as well) by extracting the $\Lambda$ dependence from the implicit regularization function. In this way, one can very easily extend the applicability of the model to larger values of $\mu$ by allowing a running $G(\Lambda)$ and a $\mu$ dependence for $\Lambda$.

For the density dependence of $\Lambda$, as said above, there is a great deal of arbitrariness. As discussed, one could fix this dependence, for instance, by matching the density dependence of $G(\Lambda) \Lambda^{2}$ with the prediction of perturbative QCD for the running of the QCD coupling constant $\alpha_{s}$ at high densities - the one-loop prediction is that the coupling decreases logarithmically with $\mu$ for large values of $\mu$. However, for our purposes here of showing the qualitative results only, we use the simple formula for $\mu \geqslant \mu_{0}=235 \mathrm{MeV}$,

$$
\Lambda=\Lambda_{0}\left[1+\alpha \log \left(\frac{\mu}{\mu_{0}}\right)\right]
$$

where $\alpha$ is a constant. When this is used in the expression for $G(\Lambda) \Lambda^{2}$, one obtains that $G(\Lambda) \Lambda^{2}$ decreases logarithmically with $\mu$, mocking up in a rather crude way the prediction of perturbative QCD for the running of $\alpha_{s}$ with $\mu$. In Fig. 4, we show the numerical results $\Delta$ as a function of $\mu$ for different values of $\alpha$. We use for the diquark pairing strength the value $G=3.1 \mathrm{GeV}^{-2}$. The other parameters are $M_{0}=312 \mathrm{MeV}$ and $\Lambda_{0}=932 \mathrm{MeV}$, which are the values obtained in vacuum for $f_{\pi}=93 \mathrm{MeV}$ and $\langle\bar{\psi} \psi\rangle_{0}=(-250 \mathrm{MeV})^{3}$. The results show the expected behavior of the gap growing faster with $\mu$ as $\Lambda$ increases with $\mu$, i.e., as $\alpha$ increases.

For completeness, we include the effects of temperature. We calculate the critical temperature $T_{c}$ above which $\Delta=0$, for different values of $\mu$. We rewrite Eq. (26) for $\Delta=0$ including the effect of temperature

$$
\begin{aligned}
1= & 16 G \int \frac{d^{4} k}{(2 \pi)^{4}} \frac{i}{k_{0}^{2}-(k+\mu)^{2}} \tanh \left(\frac{\beta}{2}|k+\mu|\right) \\
& +(\mu \rightarrow-\mu),
\end{aligned}
$$

where

$$
\tanh \left(\frac{\beta}{2}|k \pm \mu|\right)=1-2 n_{ \pm}^{\prime}(k)
$$

with

$$
n_{ \pm}^{\prime}(k)=\frac{1}{e^{\beta|k \pm \mu|}+1} .
$$

Using the same manipulations to isolate the divergent integrals as explained before and after some algebraic effort, we can write

$$
\begin{aligned}
\frac{1}{4 G}= & \Lambda^{2}\left\{-\frac{2}{3} \frac{\langle\bar{\psi} \psi\rangle_{0}}{M_{0} \Lambda_{0}^{2}}+\frac{2}{3}\left(\frac{M_{0}^{2}}{\Lambda_{0}^{2}}+\frac{2 \mu^{2}}{\Lambda^{2}}\right) \frac{f_{\pi}^{2}}{M_{0}^{2}}\right. \\
& \left.+\frac{1}{2 \pi^{2}}\left[\frac{M_{0}^{2}}{\Lambda_{0}^{2}}-\frac{3 \mu^{2}}{\Lambda^{2}}-\frac{2 \mu^{2}}{\Lambda^{2}} \ln \left(\frac{\mu^{2}}{\Lambda^{2}} \frac{\Lambda_{0}^{2}}{M_{0}^{2}}\right)\right]\right\} \\
& +Q(\mu, T)+Q(-\mu, T),
\end{aligned}
$$

where

$$
\begin{aligned}
Q(\mu, T)= & -\frac{2}{\pi^{2}} \int_{0}^{\infty} d k \frac{k^{2}}{\left(k^{2}+\mu^{2}\right)^{1 / 2}}\left[n_{+}^{\prime}(k)+n_{-}^{\prime}(k)\right] \\
& +\frac{2 \mu}{\pi^{2}} \int_{0}^{\infty} d k \frac{k^{3}}{\left(k^{2}+\mu^{2}\right)^{3 / 2}}\left[n_{+}^{\prime}(k)-n_{-}^{\prime}(k)\right] \\
& -\frac{3 \mu^{2}}{\pi^{2}} \int_{0}^{\infty} d k \frac{k^{4}}{\left(k^{2}+\mu^{2}\right)^{5 / 2}}\left[n_{+}^{\prime}(k)+n_{-}^{\prime}(k)\right] \\
& +\frac{1}{2 \pi^{2}} \int_{0}^{\infty} d k k^{2} I(k) \tanh \left(\frac{\beta}{2}|k+\mu|\right),
\end{aligned}
$$

with

$$
I(k)=\frac{2 k^{5}+5 k^{2} \mu^{2}(k-\mu)-2 \mu^{5}-2\left(k^{2}+\mu^{2}\right)^{5 / 2}}{\left(k^{2}+\mu^{2}\right)^{5 / 2}(k-\mu)}
$$

In Fig. 5, we plot the critical temperature as function of $\mu$. In the solid line, we have kept the coupling $G$ fixed; and in the dashed and dotted lines, the coupling is a function of $\mu$ through the $\mu$ dependence of $\Lambda$ as in Eq. (28). As seen, the critical temperature increases with $\mu$, and this increase is faster 


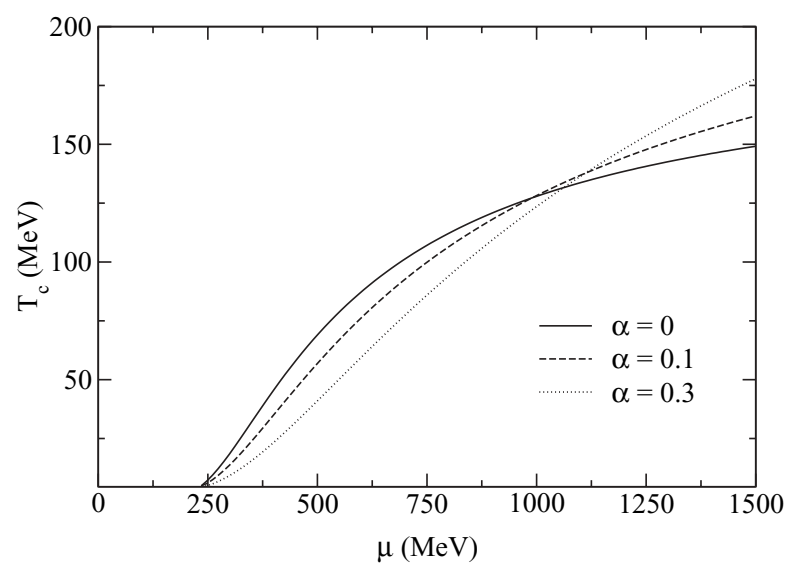

FIG. 5. Critical temperature for the superconducting gap as a function of $\mu$ for different values of $\alpha$ in Eq. (28).

as $\alpha$ increases for large $\mu$. The values of $T_{c}$ obtained within this scheme seem a little larger than those obtained with cutoff regularization.

\section{CONCLUSIONS AND PERSPECTIVES}

We have considered an extension of the NJL model to high densities and temperatures as proposed in Ref. [11] and used an alternative strategy to handle ultraviolet divergences. This extension is implemented by allowing the regularization scale $\Lambda$ to increase at high densities with the simultaneous decrease of the coupling $G$. Making use of the scaling relations of Eqs. (8) and (9), and the definitions in Eqs. (15) and (16), the two one-loop divergent integrals $I_{\text {quad }}$ and $I_{\log }$ at scales $\Lambda_{0}$ and $M_{0}$ can be related to the same $I_{\text {quad }}$ and $I_{\log }$ at different scales $\Lambda$ and $M$.
Although our numerical results are in qualitative agreement with those of Casalbuoni et al. [11] for the $\mu$ dependence of the superconducting gap $\Delta$, we believe the present approach is different from their results in several aspects. Perhaps the most important one is that no specific regularization distribution was used to obtain the running of $G$ with $\Lambda$. Proceeding this way made it possible to circumvent many troubles commonly found when traditional regularization schemes are used, such as those based on a three- or four-momentum cutoff or PauliVillars and proper-time regularizations, leading in general to global and gauge symmetry violations, and to the breaking of causality and unitarity. Although in some circumstances such aspects might not be of crucial importance, in many others, such as in hadronic correlation functions, remarkable differences appear [41]. Among them, only physical thresholds (independent of $\Lambda$ ) are present, allowing thus the preservation of unitarity. In addition, it is easy to show that causality is also preserved by checking that the amplitudes obey the correct dispersion relations. Gauge symmetry is also preserved, and the ambiguities are eliminated at the one-loop level, as shown in Ref. [23] in the context of the gauged NJL model. These matters are discussed in detail in a separate publication [25].

Another interesting aspect of the present approach is that it can be used with heavy quarks. The scaling relations involve the ratio of the quark mass to the implicit regulation scale and naturally take into account short-distance effects as the quark mass increases. This is very important in connection with studies of heavy-quark bound states in highly excited quark matter.

\section{ACKNOWLEDGMENTS}

This work was partially supported by the Brazilian agencies CAPES, CNPq, FAPERJ, and FAPESP.
[1] Y. Nambu and G. Jona-Lasinio, Phys. Rev. 122, 345 (1961); 124, 246 (1961).

[2] T. Hatsuda and T. Kunihiro, Phys. Lett. B145, 7 (1984); Phys. Rev. Lett. 55, 158 (1985); Prog. Theor. Phys. 74, 765 (1985).

[3] V. Bernard, Ulf-G. Meissner, and I. Zahed, Phys. Rev. Lett. 59, 966 (1987); Phys. Rev. D 36, 819 (1987).

[4] U. Vogl and W. Weise, Prog. Part. Nucl. Phys. 27, 195 (1991).

[5] S. P. Klevansky, Rev. Mod. Phys. 64, 649 (1992).

[6] T. Hatsuda and T. Kunihiro, Phys. Rep. 247, 221 (1994).

[7] Chr.V. Christov, A. Blotz, H.-C. Kim, P. Pobylitsa, T. Watabe, T. Meissner, E. Ruiz Arriola, and K. Goeke, Prog. Part. Nucl. Phys. 37, 91 (1996).

[8] J. Bijnens, Phys. Rep. 265, 369 (1996).

[9] V. Bernard, A. H. Blin, B. Hiller, Y. P. Ivanov, A. A. Osipov, and U.-G. Meissner, Ann. Phys. (NY) 249, 499 (1996).

[10] G. Ripka, Quarks Bound by Chiral Fields (Oxford Science Publications, London, 1997).

[11] R. Casalbuoni, R. Gatto, G. Nardulli, and M. Ruggieri, Phys. Rev. D 68, 034024 (2003).

[12] F. Gastineau, R. Nebauer, and J. Aichelin, Phys. Rev. C 65, 045204 (2002).

[13] T. M. Schwarz, S. P. Klevansky, and G. Papp, Phys. Rev. C 60, 055205 (1999).
[14] R. L. S. Farias, G. Dallabona, G. Krein, and O. A. Battistel, Phys. Rev. C 73, 018201 (2006).

[15] V. Bernard, R. L. Jaffe, and U.-G. Meissner, Nucl. Phys. B308, 753 (1988)

[16] Bing He, Hu Li, C. M. Shakin, and Qing Sun, Phys. Rev. C 67, 065203 (2003).

[17] W. Broniowski, G. Ripka, E. Nikolov, and K. Goeke, Z. Phys. A 354, 421 (1996).

[18] F. Doring, A. Blotz, C. Schuren, T. Meissner, E. Ruiz Arriola, and K. Goeke, Nucl. Phys. A536, 548 (1992).

[19] R. M. Davidson and E. Ruiz Arriola, Phys. Lett. B359, 273 (1995); B348, 163 (1995).

[20] O. A. Battistel, Ph.D. thesis, Universidade Federal de Minas Gerais, Brazil, 1999.

[21] O. A. Battistel and G. Dallabona, Nucl. Phys. B610, 316 (2001); Phys. Rev. D 65, 125017 (2002); J. Phys. G 28, 2539 (2002).

[22] D. E. Carneiro, A. P. Baeta Scarpelli, M. Sampaio, and M. C. Nemes, J. High Energy Phys. 12 (2003) 44.

[23] O. A. Battistel and M. C. Nemes, Phys. Rev. D 59, 055010 (1999).

[24] O. A. Battistel and G. Krein, Mod. Phys. Lett. A A18, 2255 (2003). 
[25] O. A. Battistel, G. Dallabona, and G. Krein, Phys. Rev. D 77, 065025 (2008).

[26] O. A. Battistel and G. Dallabona, Eur. Phys. J. C 45, 721 (2006).

[27] D. Blaschke, Yu. L. Kalinovski, P. Petrow, S. M. Schmidt, M. Jaminon, and B. Van den Bossche, Nucl. Phys. A592, 561 (1995).

[28] P. Costa, M. C. Ruivo, and Yu. L. Kalinovsky, Phys. Lett. B577, 129 (2003).

[29] Claudia Ratti, Michael A. Thaler, and Wolfram Weise, Phys. Rev. D 73, 014019 (2006).

[30] O. A. Battistel and G. Dallabona, Phys. Rev. D 72, 045009 (2005).
[31] K. Rajagopal and F. Wilczek, in Handbook of $Q C D$, edited by M. Shifman (World Scientific, Singapore, 2001).

[32] M. G. Alford, Annu. Rev. Nucl. Part. Sci. 51, 131 (2001).

[33] I. A. Shovkovy, Found. Phys. 35,1309 (2005).

[34] G. Nardulli, Riv. Nuovo Cim. 25N3, 1 (2002).

[35] D. H. Rischke, Prog. Part. Nucl. Phys. 52, 197 (2004).

[36] M. Buballa, Phys. Rep. 407, 205 (2005).

[37] M. Huang, Int. J. Mod. Phys. E 14, 675 (2005).

[38] T. Schäfer, hep-ph/0509068.

[39] A. W. Steiner, S. Reddy, and M. Prakash, Phys. Rev. D 66, 094007 (2002).

[40] D. T. Son, Phys. Rev. D 59, 094019 (1999).

[41] R. L. S. Farias, G. Krein, and O. A. Battistel, AIP Conf. Proc. 739, 431 (2005). 